\title{
Involvement of the Olfactory Apparatus by Gliomas
}

\author{
(D) X. Wu, (D)Y. Li, (D)C.M. Glastonbury, and DS. Cha
}

\begin{abstract}
SUMMARY: The olfactory bulbs and tracts are central nervous system white matter tracts maintained by central neuroglia. Although rare, gliomas can originate from and progress to involve the olfactory apparatus. Through a Health Insurance Portability and Accountability Act-compliant retrospective review of the institutional teaching files and brain MR imaging reports spanning 10 years, we identified 12 cases of gliomas involving the olfactory bulbs and tracts, including 6 cases of glioblastoma, 2 cases of anaplastic oligodendroglioma, and 1 case each of pilocytic astrocytoma, diffuse (grade II) astrocytoma, anaplastic astrocytoma (grade III), and diffuse midline glioma. All except the pilocytic astrocytoma occurred in patients with known primary glial tumors elsewhere. Imaging findings of olfactory tumor involvement ranged from well-demarcated enhancing masses to ill-defined enhancing infiltrative lesions to nonenhancing masslike FLAIR signal abnormality within the olfactory tracts. Familiarity with the imaging findings of glioma involvement of the olfactory nerves is important for timely diagnosis and treatment of recurrent gliomas and to distinguish them from other disease processes.
\end{abstract}

ABBREVIATIONS: GBM = glioblastoma multiforme; TMZ = temozolomide; EGFR = epidermal growth factor receptor; IDH1 = Isocitrate dehydrogenase 1; MGMT = O6-methylguanine methyltransferase

T he olfactory bulbs and tracts are central nervous system white matter tracts extending directly to the cerebrum, maintained by a combination of specialized olfactory ensheathing cells and central neuroglia, including astrocytes and oligodendrocytes. ${ }^{1}$ As a result, gliomas can rarely originate from and progress to involve the olfactory apparatus. Relatively common neoplasms that involve the olfactory grooves include meningiomas arising from the anterior cranial fossa dura and sinonasal malignancies extending intracranially such as squamous cell carcinoma and esthesioneuroblastoma. ${ }^{2}$ These entities have characteristic imaging findings, and the purpose of this article is to characterize the range of imaging findings of gliomas that affect the olfactory

Received November 7, 2019; accepted after revision January 25, 2020.

From the Department of Radiology and Imaging Sciences (X.W.), Emory University, Atlanta, Georgia; Departments of Clinical Radiology (Y.L., C.M.G.), Otolaryngology Head and Neck Surgery (C.M.G.), Radiation Oncology (C.M.G.), Radiology (S.C.), and Neurological Surgery (S.C.), University of California, San Francisco, San Francisco, California.

Drs Glastonbury and Cha contributed equally to this work.

Paper previously presented at: Annual Meeting of the American Society of Neuroradiology and the Foundation of the ASNR Symposium, June 2-7, 2018; Vancouver, British Columbia, Canada.

Please address correspondence to Xin Wu, MD, Department of Radiology and Imaging Science, Emory University, 1364 Clifton Rd NE, Suite BG 20, Atlanta, GA 30322; e-mail: xin.wu@emory.edu; @CynXinWu

Indicates article with supplemental on-line table.

http://dx.doi.org/10.3174/ajnr.A6471 bulbs and tracts and to differentiate them from other masses of the anterior cranial fossa.

\section{Case Series}

A Health Insurance Portability and Accountability Act-compliant retrospective search through University of California San Francisco radiology institutional teaching files and radiology reports for patients with olfactory masses was conducted, with an inclusion criterion of a known history of glioma or pathologically proved glial neoplastic involvement of the olfactory nerve. Twelve patients ( 3 women and 9 men) were identified with glial masses involving the olfactory bulbs. Patient ages ranged from 10 to 74 years (On-line Table). A single case of primary pilocytic astrocytoma originating from the olfactory bulb was identified. The remaining 11 patients had known pre-existing glial tumors elsewhere, with multicentric or progressive involvement of the olfactory bulb/tract. There were 6 cases of glioblastomas, 2 cases of anaplastic oligodendrogliomas, and 1 case each of diffuse astrocytoma, anaplastic astrocytoma, and diffuse midline glioma.

Case 1. A 27-year-old man presented with mild hyperprolactinemia and underwent a dedicated pituitary and sella protocol MR imaging, on which a well-circumscribed uniformly enhancing mass was seen to arise from the left olfactory groove, with mild surrounding dural thickening and enhancement (Fig 1). There 
was no tumor extension through the cribriform plate, and no other intracranial lesions were identified on follow-up brain imaging. On further questioning, the patient revealed partial anosmia. The radiology interpretation favored a diagnosis of meningioma, and the operative note even indicated a dural tail associated with the mass, which was inseparable from the left olfactory nerve. The final pathologic diagnosis yielded pilocytic astrocytoma, World Health Organization grade I.

Case 2. A 51-year-old man with a history of left frontal lobe grade II astrocytoma treated 12 years prior by surgical resection and temozolomide (TMZ) was found to have nonenhancing masslike T2-weighted/FLAIR signal abnormality in the olfactory nerve ipsilateral to his original tumor, concerning for slowly progressive recurrence.

Case 3. A 46-year-old woman was originally diagnosed with grade III diffuse anaplastic oligodendroglioma of the left inferior frontal lobe, treated with resection and chemoradiation. She presented to our institution 8 years after the initial treatment for continued follow-up. During the subsequent 8 years, the tumor demonstrated subtle and slow progression during multiple examinations, as manifested by progressive nonenhancing masslike FLAIR signal abnormality involving the left anterior perforated substance and olfactory nerve (Fig 2).
Case 4. A 53-year-old male patient with a right temporal anaplastic oligodendroglioma had undergone subtotal resection 7 years prior followed by chemoradiation. He demonstrated slowly progressive bulky nonenhancing disease involving the olfactory cortex and olfactory bulb (Fig 3).

Case 5. A 10-year-old boy presented for continued management of his orbital rhabdomyosarcoma, originally diagnosed and treated 8 years prior. On his initial (pretreatment) imaging, a right anterior temporal mass was also identified. On further work-up and biopsy, this tumor was found to represent a grade III anaplastic astrocytoma. Abnormal nonenhancing nodular T2 hyperintensity was present along the olfactory nerve, though there was no definite involvement of the ipsilateral olfactory cortex (Fig 4). Signal abnormality was also seen in the bilateral internal auditory canals and along the expected course of the left abducens nerve, likely due to leptomeningeal disease spread.

Case 6. A 15-year-old male patient presented with diffuse midline glioma diagnosed 2 years prior, when he underwent treatment with chemoradiation. One year after the diagnosis, additional treatment was required with radiation, nivolumab, and bevacizumab. Imaging demonstrated diffuse nonenhancing T2/FLAIR signal abnormality of the bilateral frontotemporal lobes and olfactory nerves (Fig 5).
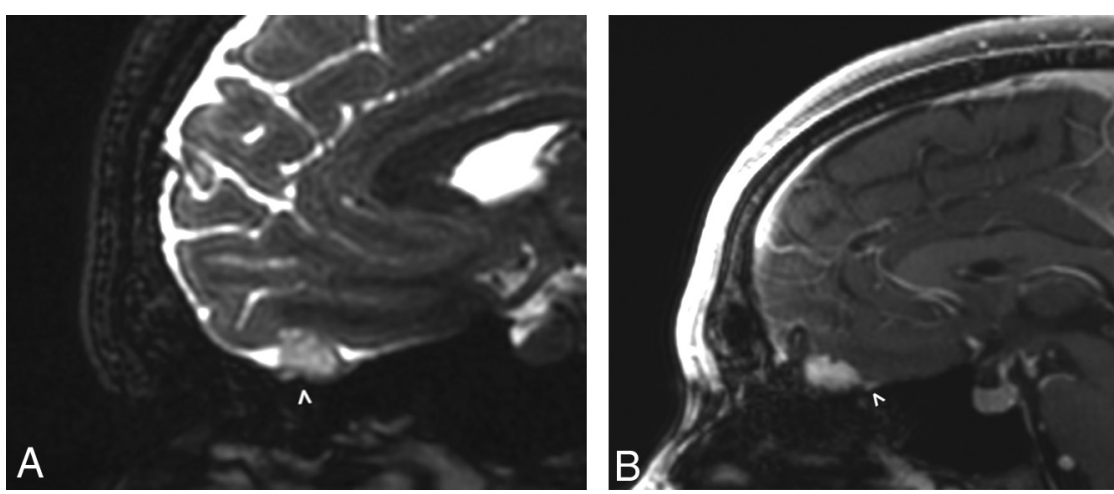

FIG 1. A 27-year-old man with mild hyperprolactinemia (case 1). Sagittal FIESTA (A) demonstrates a $T 2$ hyperintense apparent extra-axial mass (arrowhead) within the left anterior cranial fossa. On the sagittal postcontrast T1-weighted image $(B)$, the mass has uniform enhancement with a subtle adjacent dural tail (arrowhead). On imaging, this was believed to be a meningioma, but at resection, it was determined to be a pilocytic astrocytoma.
Case 7. A 61-year-old woman originally presented with a right temporal lobe mass diagnosed as Isocitrate dehydrogenase 1 (IDH1) $\mathrm{R} 132 \mathrm{H}$ wild-type, epidermal growth factor receptor (EGFR)-amplified, O6-methylguanine methyltransferase (MGMT) unmethylated glioblastoma multiforme (GBM), which underwent partial resection, radiation, and TMZ therapy. A year later, there was a new nodular enhancing mass within the surgical bed compatible with disease progression. The contralateral olfactory nerve also demonstrated progressive masslike FLAIR signal abnormality as well as enhancement, compatible with multicentric disease recurrence and progression.
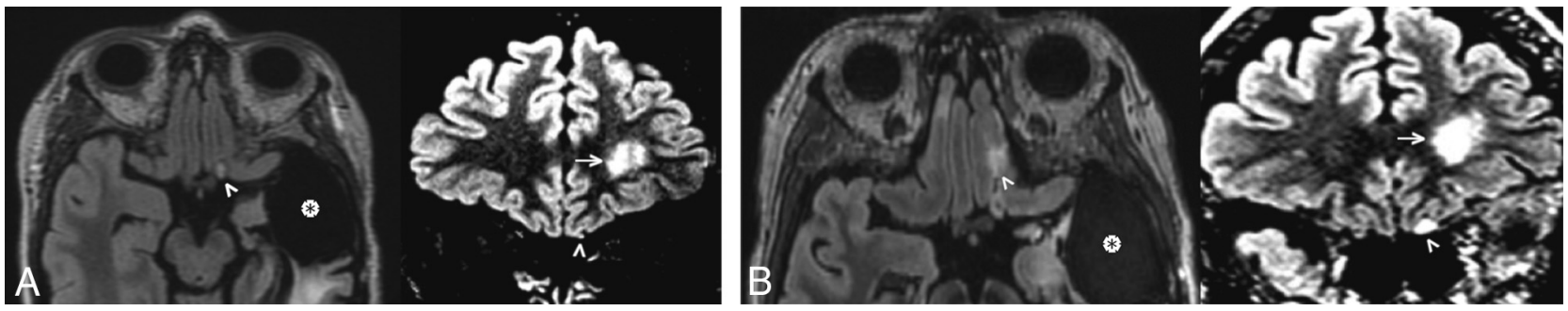

FIG 2. A 46-year-old woman with left inferior frontal grade III diffuse anaplastic oligodendroglioma previously treated with resection and chemoradiation (case 3). Axial and coronal FLAIR images obtained 8 years after the initial treatment $(A)$ demonstrate a left temporal resection cavity (asterisk) and unchanged FLAIR signal in the left frontal lobe (arrow). There is subtle nodular FLAIR signal abnormality (arrowheads) of the left olfactory bulb. Axial and coronal FLAIR images obtained 16 years after the initial treatment $(B)$ show minimal change around the resection cavity (asterisk) but progressive enlargement of the hyperintense left olfactory bulb (arrowheads). 

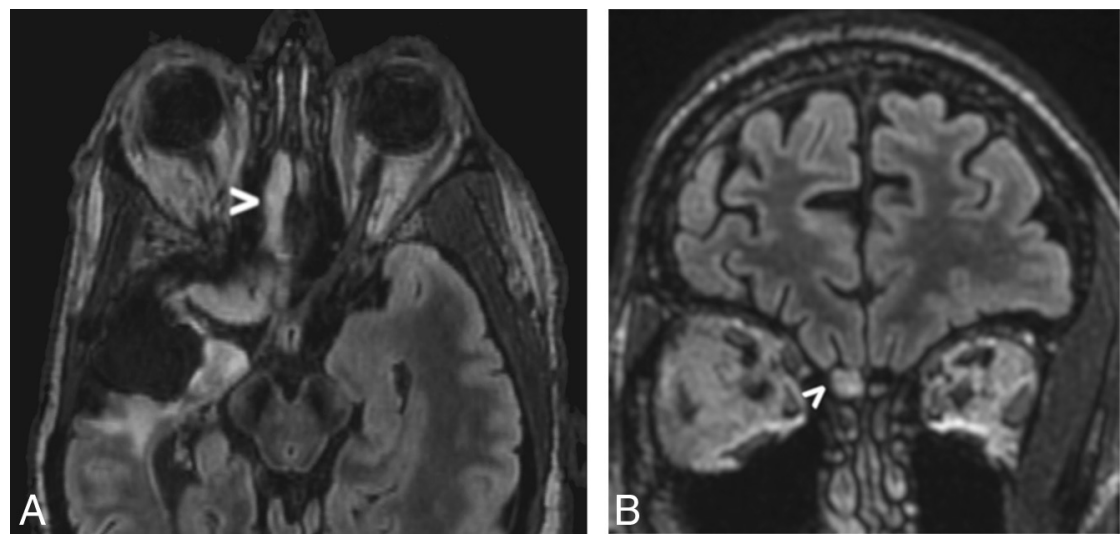

FIG 3. A 53-year-old man with a right temporal anaplastic oligodendroglioma previously treated with subtotal resection and chemoradiation (case 4). Axial FLAIR image (A) obtained 7 years after the initial treatment demonstrates masslike FLAIR signal abnormality within the right olfactory cortex as well as the right olfactory tract and bulb (arrowhead), compatible with oligodendroglioma involvement. Coronal FLAIR image $(B)$ obtained at the same time illustrates an asymmetric masslike FLAIR signal abnormality within the right olfactory tract and bulb (arrowhead), while the contralateral olfactory bulb remains normal in caliber.
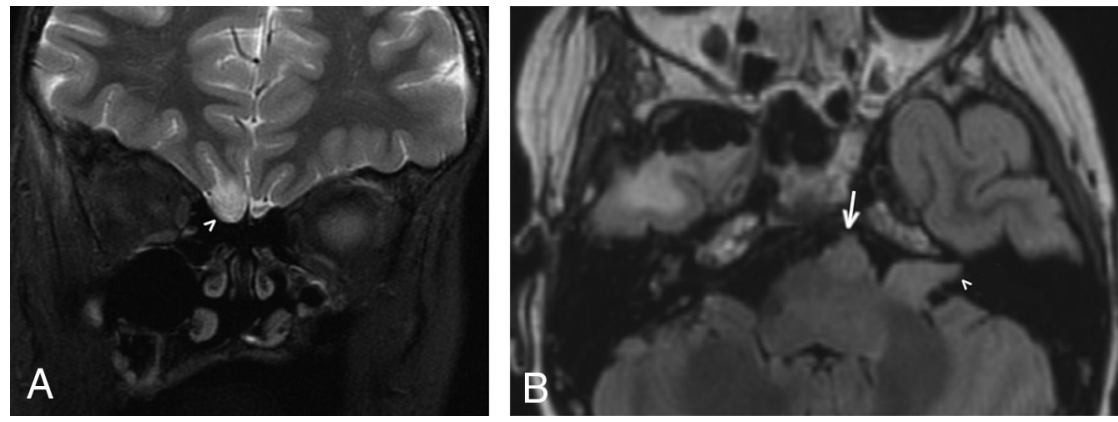

FIG 4. A 10-year-old boy with right anterior temporal grade III anaplastic astrocytoma detected 8 years prior (case 5). Coronal FLAIR image $(A)$ demonstrates a nonenhancing masslike signal abnormality within the right olfactory tract (arrowhead), while an axial FLAIR image (B) obtained at the same time demonstrates a nonenhancing masslike signal abnormality within the left internal auditory canal (arrowhead) as well as in the prepontine cistern, along the presumed location of the left abducens nerve/CN VI (arrow). Combined, findings are compatible with leptomeningeal disease spread involving multiple cranial nerves.

Case 8. A 69-year-old woman presented with a large left frontal IDH1 R132H wild-type, MGMT-unmethylated, and EGFRunamplified GBM with sarcomatous features. She underwent radiation therapy with concurrent TMZ. When the tumor progressed, she underwent adjuvant TMZ followed by a MEDI4736 vaccine trial and bevacizumab. A nodular enhancing mass developed in the left olfactory groove during treatment, inseparable from the olfactory nerve.

Case 9. A 74-year-old man presented with a right frontal GBM, which did not involve the olfactory cortex. He was then found to have an enhancing nodular mass involving the contralateral olfactory nerve compatible with progressive disease.

Case 10. A 37-year-old man presented with a nonenhancing left olfactory nerve mass and a history of GBM centered in the right frontal operculum, resected 4 years prior (Fig 6). The mass was resected and was demonstrated to be glioblastoma.

Case 11. A 36-year-old man with an IDH1 wild-type, p53-positive, phosphatase and tensin homolog-intact, EGFRnegative GBM centered in the corpus callosum underwent treatment with radiation and TMZ. One year later, he presented with abnormal enlargement and patchy enhancement in the left olfactory nerve accompanied by extensive masslike FLAIR signal abnormality in the ipsilateral olfactory cortex. There was progressive worsening of the masslike signal abnormality and enhancement within the left olfactory nerve during studies spanning the next 3 months (Fig 7).

Case 12. A 31-year-old man was originally diagnosed with right frontal lobe grade III anaplastic astrocytoma 6 years prior. On presentation to our institution, the tumor had undergone transformation to an IDH1-mutant, EGFR-negative, MGMT-methylated GBM, which was 1p19q-codeleted. There was bulky nonenhancing signal abnormality in the right gyrus rectus, orbitofrontal gyrus, anterior temporal lobe, and olfactory nerve, compatible with progressive disease involvement (Fig 8).

\section{DISCUSSION}

The first cranial nerve (CN I), also known as the olfactory nerve, is a purely sensory nerve that supplies the sensation of smell via specialized olfactory sensory neurons that arise from the nasal mucosa of the superior nasal cavity. Extending through the cribriform plate, small fibers of unmyelinated bipolar olfactory nerve cells synapse with secondary mitral and tufted neurons in the olfactory bulbs. Also known as projection neurons, these mitral and tufted neurons form myelinated olfactory tracts that lie within the bony olfactory sulcus and groove in the medial-most portions of the floor of the anterior cranial fossa. The olfactory tracts directly project to the olfactory cortices bilaterally, which include the posterior orbitofrontal gyrus, amygdala, and uncus, where the sensation of smell is synthesized and processed. ${ }^{3,4}$

Our study of 12 patients with glioma involving the olfactory bulbs and tracts highlights an uncommon-but-clinically important phenomenon. All except 1 of the cases represented secondary involvement through direct infiltration of tumor or multifocal/ multicentric disease in patients with known glioma diagnoses, including anaplastic oligodendroglioma, diffuse and anaplastic 

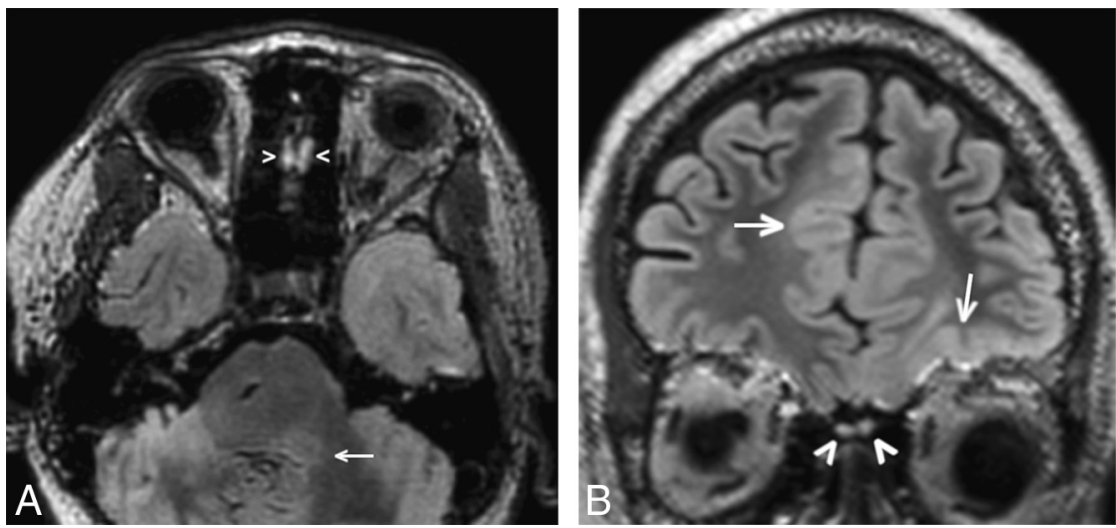

FIG 5. A 15-year-old boy presenting for follow-up of diffuse midline glioma initially diagnosed 2 years earlier and treated with chemoradiation (case 6). An axial FLAIR image (A) demonstrates nonenhancing T2/FLAIR signal abnormality of the bilateral olfactory nerves (arrowheads) and left dorsal pons (arrow), as well as cortical thickening and signal abnormality in the left anterior temporal lobe. Coronal FLAIR image (B) illustrates thickening and FLAIR signal abnormality involving the bilateral olfactory nerves (arrowheads) as well as multiple cortical areas in the bilateral frontal lobes (arrows). Findings are compatible with multifocal glioma involvement.

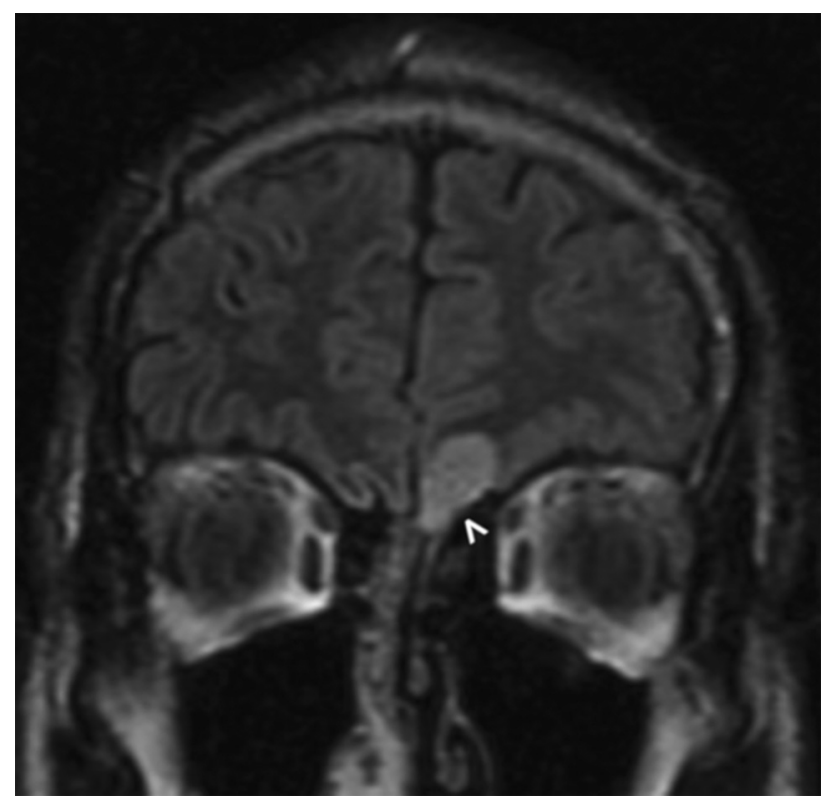

FIG 6. A 37-year-old man with a history of right frontal operculum GBM resected 4 years prior (case 10). Coronal FLAIR image demonstrates masslike FLAIR signal abnormality in the left olfactory nerve and surrounding left inferior frontal lobe (arrowhead), pathologically proved to reflect disease recurrence on resection.

astrocytomas, diffuse midline glioma, and GBM. None of the gliomas in our series demonstrated osseous destruction, extension through the skull base, or involvement of the olfactory recess. The lack of these features distinguishes these tumors radiographically from other aggressive lesions common to this site, including enthesioneuroblastoma, aggressive primary osseous lesions, and intracranial extension of primary head and neck neoplasms, such as squamous cell carcinoma. ${ }^{5,6}$

In all patients with GBM, olfactory bulb and tract involvement represented progression of previously treated disease elsewhere in the cerebrum. In 3 cases, the olfactory nerve affected was located contralateral to the site of the originally treated tumor, compatible with the underlying multifocal/infiltrative nature of GBM. From animal studies, there is evidence that GBM cancer-initiating cells may have special affinity for the subventricular zones and olfactory bulbs, mimicking the behavior or neural stem cells. ${ }^{7}$ This mechanism potentially explains the tropism for the olfactory system that is demonstrated by progressive gliomas in our series.

Typically, the imaging characteristics of progressive olfactory tract involvement mirrored those of the pre-existing glial tumor. In our series, 4 of the 6 patients with GBM had enhancing disease involving the olfactory bulb, while 2 patients had nonenhancing infiltrative lesions. For 4 of the 6 patients, the IDH1 genotyping was available. Of these patients, 1 patient with secondary IDH1-mutant disease demonstrated nonenhancing involvement of the olfactory nerve, while 3 patients with $I D H 1$ wild-type disease all demonstrated enhancing tumor along the olfactory nerve. These findings are compatible with the most common MR imaging appearance of $I D H 1$ wild-type versus mutant GBMs. ${ }^{8}$

In 1 case with a pre-existing glial tumor, there was widespread intracranial disease, including involvement of multiple cranial nerves, raising suspicion for leptomeningeal spread of disease, which resulted in the signal abnormality involving the olfactory nerve. However, in 6 of the remaining 10 progressive cases, there was concurrent or prior involvement of ipsilateral olfactory cortex by tumor, supporting the hypothesis of direct tumor progression through parenchymal glial tracts.

The sole case of primary glioma arising from the olfactory bulbs in our series was a pilocytic astrocytoma, which mimicked a meningioma on imaging as well as during surgical resection. While often seen in the setting of meningiomas, dural enhancement and thickening (also known as the dural tail sign) are nonspecific and have been reported with a variety of peripherally located parenchymal lesions. ${ }^{9}$ More specifically, supratentorial pilocytic astrocytomas adjacent to the cerebral convexity and anterior clinoid process have been reported to mimic meningiomas. ${ }^{10,11}$

To our knowledge, this is the first time that a primary glial neoplasm has been reported to arise from the olfactory tract. Although a case report has previously reported olfactory groove involvement by a large pleomorphic xanthoastrocytoma, it is unclear whether the lesion originated from the inferior frontal lobe parenchyma or olfactory tracts on the basis of imaging and intraoperative findings. ${ }^{12}$ Primary ganglioglioma, which contains both glial and neuronal cells, has previously been reported to involve the olfactory cortex and olfactory nerve. ${ }^{13}$ Primary tumors arising from olfactory ensheathing cells have also been previously described. ${ }^{14}$ These 

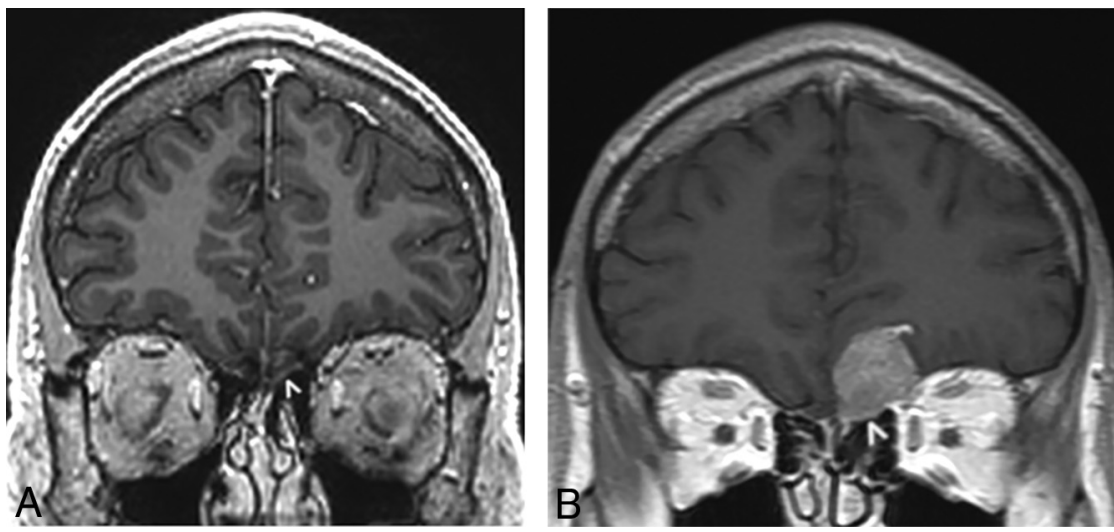

FIG 7. A 36-year-old man with a history of IDHI wild-type GBM centered in the corpus callosum, treated with radiation and TMZ (case 11). Coronal postcontrast T1-weighted echo-spoiled gradient echo image $(A)$ obtained 1 year from initial treatment demonstrates abnormal enlargement and patchy enhancement of the left olfactory nerve (arrowhead). On a follow-up coronal postcontrast T1-weighted spin-echo image obtained 3 months later $(B)$, the enhancing mass has enlarged (arrowhead) and appears to involve the leptomeningeal space, compatible with progressive recurrence.

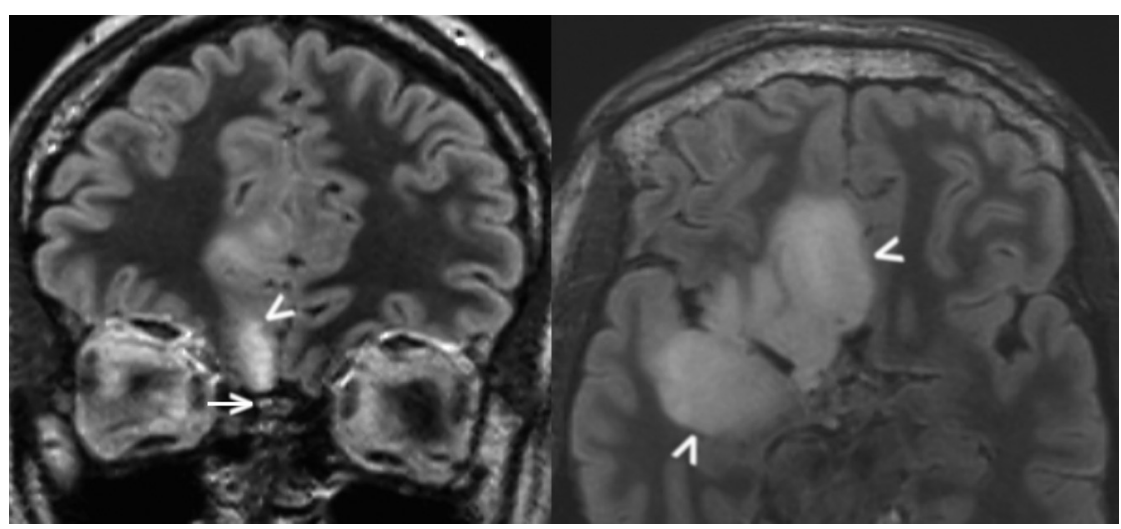

FIG 8. A 31-year-old man originally treated for right frontal lobe grade III anaplastic astrocytoma 6 years prior and now presenting with tumor transformation to an IDHI-mutant, 1p19q-codeleted GBM (case 12). Coronal and axial FLAIR images on re-presentation demonstrate a bulky nonenhancing masslike signal abnormality involving the right olfactory cortex (arrowheads) and olfactory nerve (arrow), compatible with GBM involvement.

tumors can histologically resemble olfactory groove schwannomas, which are entities of unclear origin because Schwann cells are not found in the olfactory system, though various developmental and nondevelopmental theories have been proposed to explain the origin of these tumors. ${ }^{15-17}$ These lesions can all appear similar radiologically as enhancing olfactory groove masses, which may remodel but not erode the skull base.

In all our cases, olfactory nerve involvement was detected on imaging follow-up of the patients' known gliomas or work-up for an unrelated symptom/laboratory findings without a related symptom. In at least 3 of the progressive glioma cases, abnormal signal intensity could be seen in the olfactory nerve on retrospective review of examinations performed when the finding was first reported. This highlights the importance of including the olfactory nerves in one's search pattern, because our study suggests that most patients would not present with new olfactory-related symptoms.
One of the limitations of this study is the lack of pathologic confirmation for most cases in which there was suspected involvement of the olfactory nerve by progressive glioma. In most of these cases, the ipsilateral olfactory nerve was involved or progressive disease was evident in other parts of the brain parenchyma. Thus, treatment strategies were altered on the basis of a combination of clinical and radiologic evidence of disease progression because pathologic confirmation would have demanded invasive procedures, which were not favored in risk-benefit analyses. In the single case in which a biopsy was obtained to confirm progressive disease (case 10), olfactory involvement represented the sole site of suspected recurrence and was located contralateral to the site of the previously resected tumor, thus presenting a diagnostic dilemma, which required pathologic confirmation.

\section{CONCLUSIONS}

Awareness and early detection of unusual occurrence or recurrence of gliomas involving the olfactory bulb and tract are important for diagnosis and proper clinical management. Because it can be a subtle site of tumor recurrence, the olfactory groove is an important search pattern for accurate and early diagnosis of tumor recurrence in a patient with a diagnosis of glioma, especially if the patient's tumor previously involved the olfactory cortex. Furthermore, gliomas can rarely arise from the olfactory nerve, appear discrete, and mimic meningiomas.

\section{REFERENCES}

1. Garcia-Gonzalez D, Murcia-Belmonte V, Clemente D, et al. Olfactory system and demyelination. Anat Rec (Hoboken) 2013;296:1424-34 CrossRef Medline

2. Francies O, Makalanda L, Paraskevopolous D, et al. Imaging review of the anterior skull base. Acta Radiology Open 2018;7:20584601 18776487 CrossRef Medline

3. Rea P. Olfactory Nerve. In Rea P, ed. Clinical Anatomy of the Cranial Nerves. San Diego: Academic Press; 2014:1-6

4. Som PM, Curtin HD. Head and Neck Imaging. St. Louis: Mosby; 1996

5. Dublin AB, Bobinski M. Imaging characteristics of olfactory neuroblastoma (esthesioneuroblastoma). J Neurol Surg B Skull Base 2016;77:1-5 CrossRef Medline

6. Hudgins PA, Baugnon KL. Head and neck: skull base imaging. Neurosurgery 2018;82:255-67 CrossRef Medline 
7. Kroonen J, Nassen J, Boulanger YG, et al. Human glioblastoma-initiating cells invade specifically the subventricular zones and olfactory bulbs of mice after striatal injection. Int J Cancer 2011;129:57485 CrossRef Medline

8. Yamashita K, Hiwatashi A, Togao O, et al. MR imaging-based analysis of glioblastoma multiforme: estimation of IDH1 mutation status. AJNR Am J Neuroradiol 2016;37:58-65 CrossRef Medline

9. Guermazi A, Lafitte F, Miaux Y, et al. The dural tail sign: beyond meningioma. Clin Radiology 2005;60:171-88 CrossRef Medline

10. Lee JH, Sung SK, Choi CH. Supratentorial pilocytic astrocytoma mimicking convexity meningioma with early anaplastic transformation: a case report. Brain Tumor Res Treat 2017;5:105-09 CrossRef Medline

11. Hong CS, Lehman NL, Sauvageau E. A pilocytic astrocytoma mimicking a clinoidal meningioma. Case Rep Radiology 2014;2014:524 574 CrossRef Medline
12. Kaku MV, Bhat DI, Shukla D, et al. Pleomorphic xanthoastrocytoma arising from olfactory groove: a rare location for a rare tumor. Pediatr Neurosurg 2013;49:292-96 CrossRef Medline

13. Singla N, Gupta K, Kapoor A. Ganglioglioma arising from olfactory sheath: a rare site of an uncommon tumor. J Clin Neurosci 2016;28:182-84 CrossRef Medline

14. Darie I, Riffaud L, Saikali S, et al. Olfactory ensheathing cell tumour: case report and literature review. J Neurooncol 2010;100:285-89 CrossRef Medline

15. Praharaj SS, Vajramani GV, Santosh V, et al. Solitary olfactory groove schwannoma: case report with review of the literature. Clin Neurol Neurosurg 1999;101:26-28 CrossRef Medline

16. Murakami $\mathrm{M}$, Tsukahara $\mathrm{T}$, Hatano $\mathrm{T}$, et al. Olfactory groove schwannoma: case report. Neurol Med Chir (Tokyo) 2004;44:191-94 CrossRef Medline

17. Li YP, Jiang S, Zhou PZ, et al. Solitary olfactory schwannoma without olfactory dysfunction: a new case report and literature review. Neurol Sci 2012;33:137-42 CrossRef Medline 